Supporting Information

\title{
Thulium- and Erbium-Doped Nanoparticles with \\ Poly(acrylic acid) Coating for Upconversion Cross- \\ Correlation Spectroscopy-based Sandwich \\ Immunoassays in Plasma
}

Satu Lahtinen ${ }^{\ddagger}{ }^{\dagger}{ }^{\dagger}$ Mikkel Baldtzer Liisberg ${ }^{\dagger}+$ Kirsti Raiko, $^{\dagger}$ Stefan Krause, ${ }^{*}$ Tero Soukka ${ }^{\dagger}$ and Tom Vosch

${ }^{\dagger}$ Department of Biotechnology, University of Turku, Kiinamyllynkatu 10, 20520 Turku, Finland

${ }^{+}$Nano-Science Center, Department of Chemistry, University of Copenhagen, Universitetsparken 5, 2100 Copenhagen, Denmark

${ }^{*}$ Department of Chemistry, Ludwig-Maximilians University of Munich, Butenandtstr. 5-13, 81377 Munich, Germany

$\$$ These authors contributed equally to this work

\section{Corresponding Author}

*selaht@utu.fi 


\section{Table of Contents}

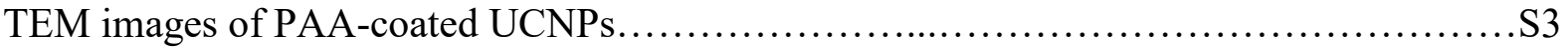

Filtration experiment of antibody conjugated UCNPs incubated in plasma.................S4

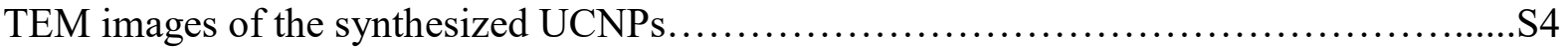

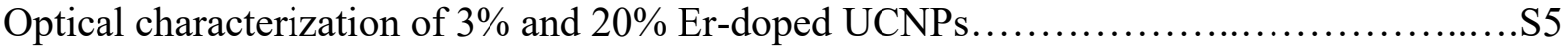

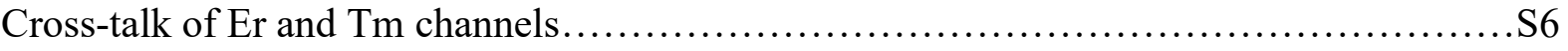

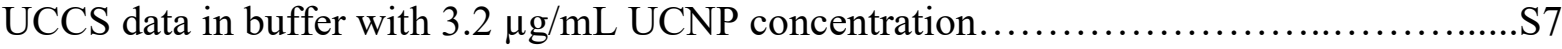

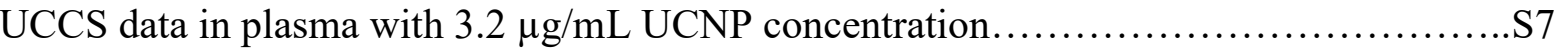

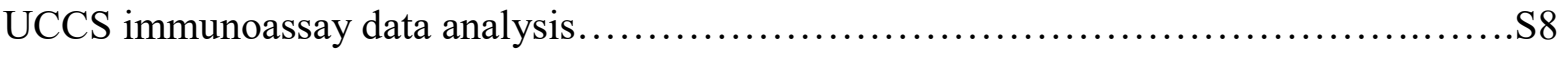

Immunoassay standard curve fitting parameters...................................... 14

Autocorrelation amplitudes in TSH immunoassay at $72 \mu \mathrm{g} / \mathrm{mL}$ UCNP concentration......S14

Autocorrelation amplitudes in TSH immunoassay at $3.2 \mu \mathrm{g} / \mathrm{mL}$ UCNP concentration.......S15

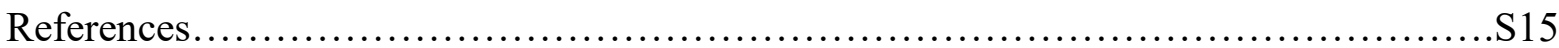



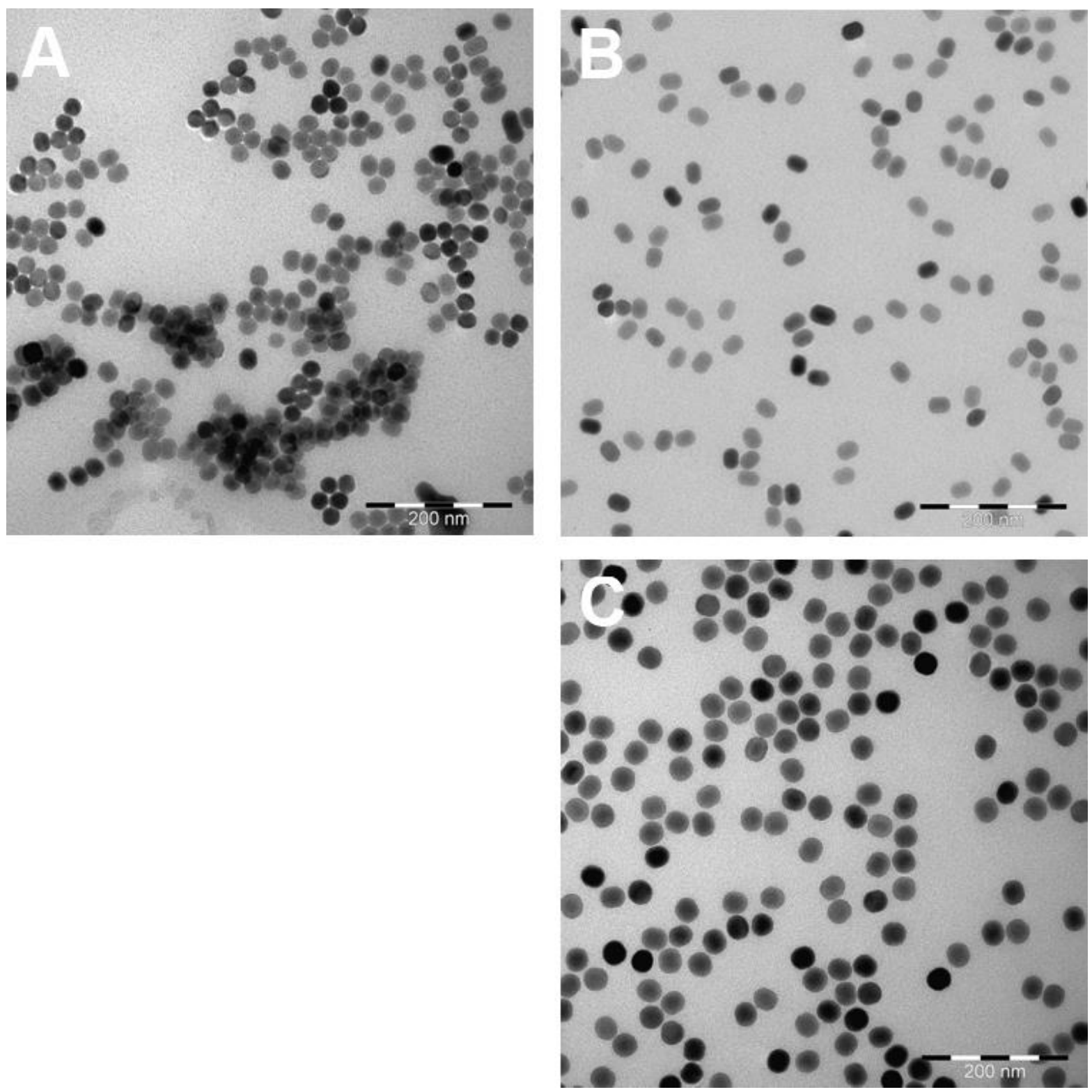

Figure S1. TEM-images of PAA-coated and antibody conjugated $\mathrm{NaYF}_{4}: \mathrm{Yb}^{3+}, \mathrm{Er}^{3+}(3 \% \mathrm{Er})$ UCNPs, which were surface modified by A) $\mathrm{HCl}$ treatment and PAA coating in water or $\mathrm{B}$ ) $\mathrm{NOBF}_{4}$ ligand exchange and PAA coating in DMF at elevated temperature, and C) PAA-coated and antibody conjugated $\mathrm{NaYF}_{4}: \mathrm{Yb}^{3+}, \mathrm{Tm}^{3+}(8 \% \mathrm{Tm})$ UCNPs, which were surface modified by using $\mathrm{NOBF}_{4}$ ligand exchange and PAA coating in DMF at elevated temperature. All the TEMsamples of antibody conjugated UCNPs have been made from water based UCNP dispersions. The scale bar is $200 \mathrm{~nm}$. 
Table S1. UCNP concentrations before and after filtrating the particles incubated in $20 \%$ plasma through different pore size filters. Standard deviations are based on six replicates.

\begin{tabular}{llcc}
\hline & & \multicolumn{2}{c}{ Surface modification } \\
\hline UCNP concentration $(\mu \mathrm{g} / \mathrm{mL})$ & non-filtered & $\mathrm{HCl}+\mathrm{PAA}$ in water & $\mathrm{NOBF}_{4}+\mathrm{PAA}$ in DMF \\
\cline { 2 - 4 } & $220 \mathrm{~nm}$ filter & $540 \pm 91$ & $200 \pm 1$ \\
\cline { 2 - 4 } & $100 \mathrm{~nm}$ filter & blocked & $190 \pm 1$ \\
\hline Total loss in concentration $(\%)$ & & $\mathbf{1 0 0}$ & $180 \pm 9$ \\
\hline
\end{tabular}

* As the $220 \mathrm{~nm}$ filter was blocked and there was no flow through, the solution could not be filtered through $100 \mathrm{~nm}$ pore size filter
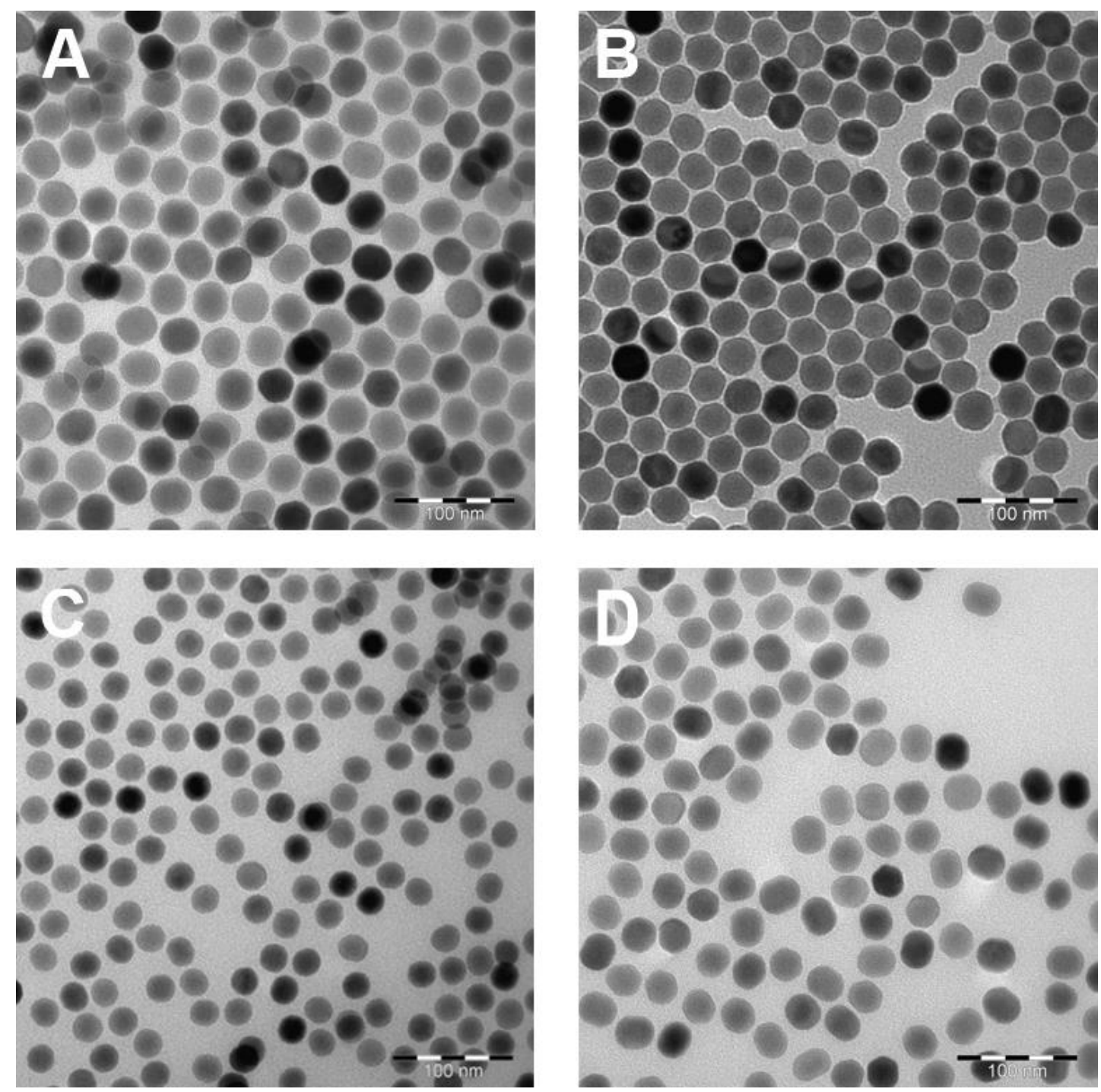

Figure S2. TEM-images of $\mathrm{NaYF}_{4}: \mathrm{Yb}^{3+}, \mathrm{Tm}^{3+} \mathrm{UCNP}$ with A) $8 \% \mathrm{Tm}$, or B) $0.5 \% \mathrm{Tm}$ (image published previously in SI of Lahtinen et al. ${ }^{1}$ ), and $\mathrm{NaYF}_{4}: \mathrm{Yb}^{3+}, \mathrm{Er}^{3+} \mathrm{UCNPs}$ with C) $20 \% \mathrm{Er}$ or D) 3\% Er image with JEM-1400 Plus TEM (80 kV electron beam, JEOL, Massaschusetts, USA). Scale bar is $100 \mathrm{~nm}$. 


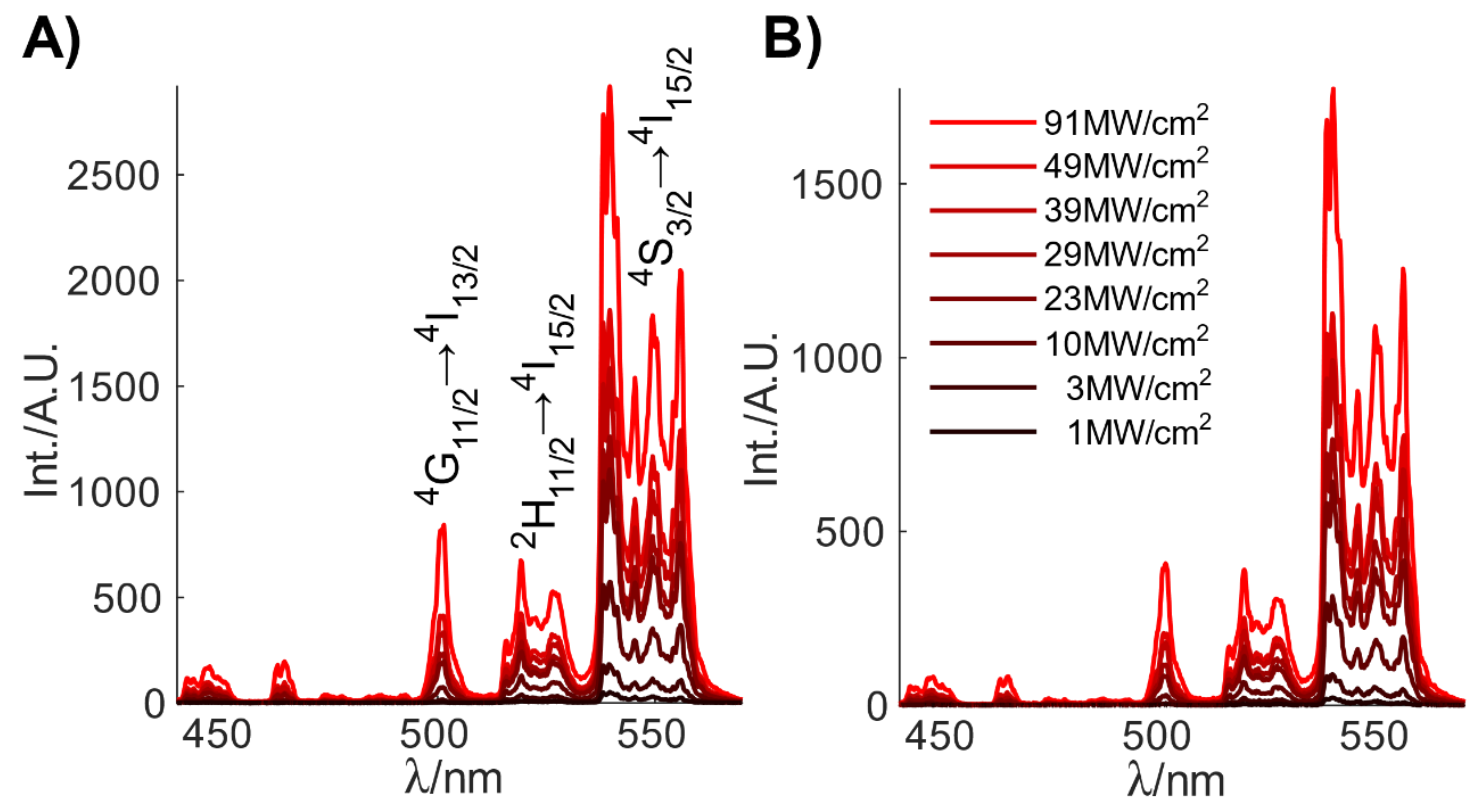

C)

D)
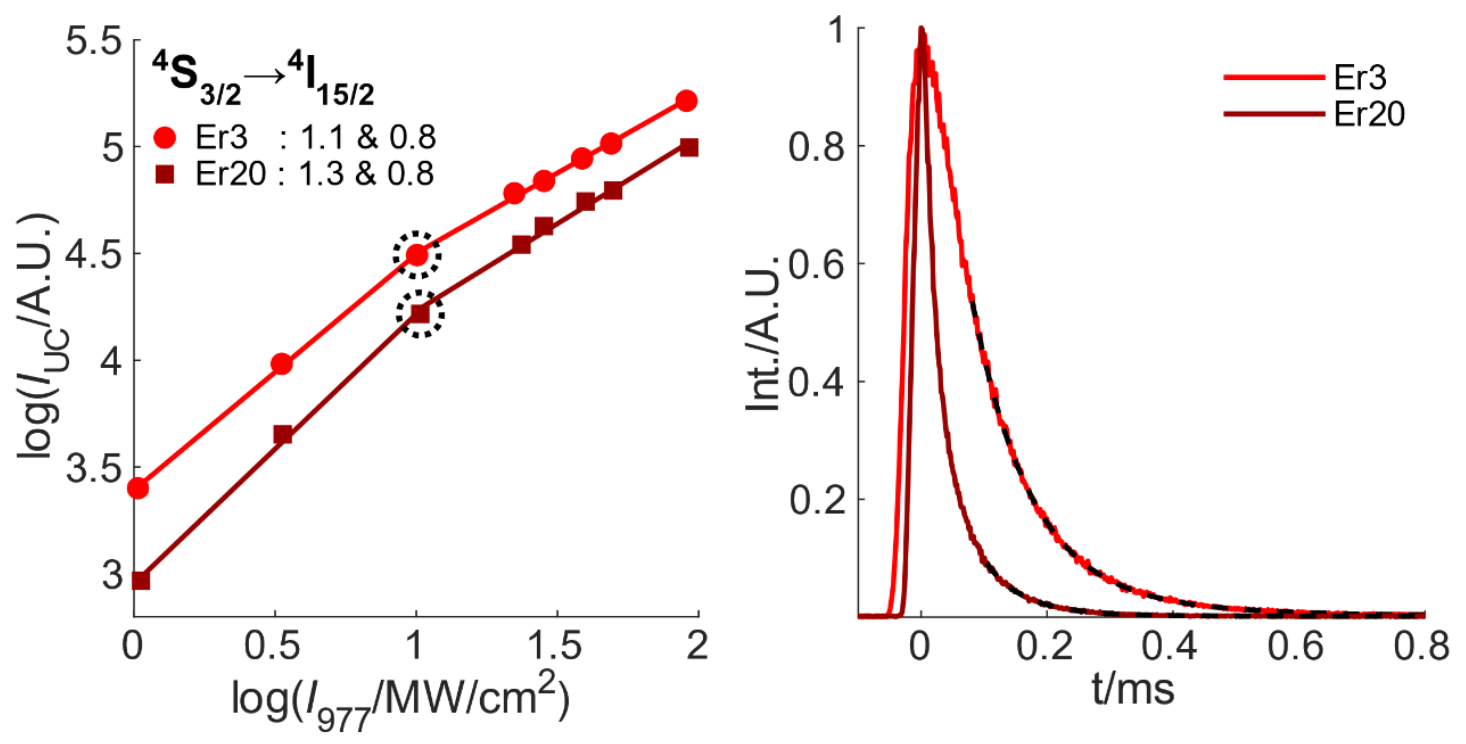

Figure S3. Optical characterization for low and high doped Er particles. Spectral power dependence of A) $\operatorname{Er}(3 \%)$ and B) $\operatorname{Er}(20 \%)$. The laser intensities used for $\operatorname{Er}(3 \%)$ are similar to those used for $\operatorname{Er}(20 \%)$ within $3 \%$. C) Power dependence of the ${ }^{4} \mathrm{~S}_{3 / 2} \rightarrow{ }^{4} \mathrm{I}_{15 / 2}$ transition for both the low and high doped Er particles. Data points marked with a dashed circle indicate transitions between different power dependent regimes for $\operatorname{Er}(3 \%)$ and $\operatorname{Er}(20 \%)$. Two different slopes are fitted for $\operatorname{Er}(3 \%)$ and $\operatorname{Er}(20 \%)$ in these high and low power regimes and noted in the figure. D) Decay time measurements of the ${ }^{4} \mathrm{~S}_{3 / 2} \rightarrow{ }^{4} \mathrm{I}_{15 / 2}$ transition for $\operatorname{Er}(3 \%)$ and $\operatorname{Er}(20 \%)$. 


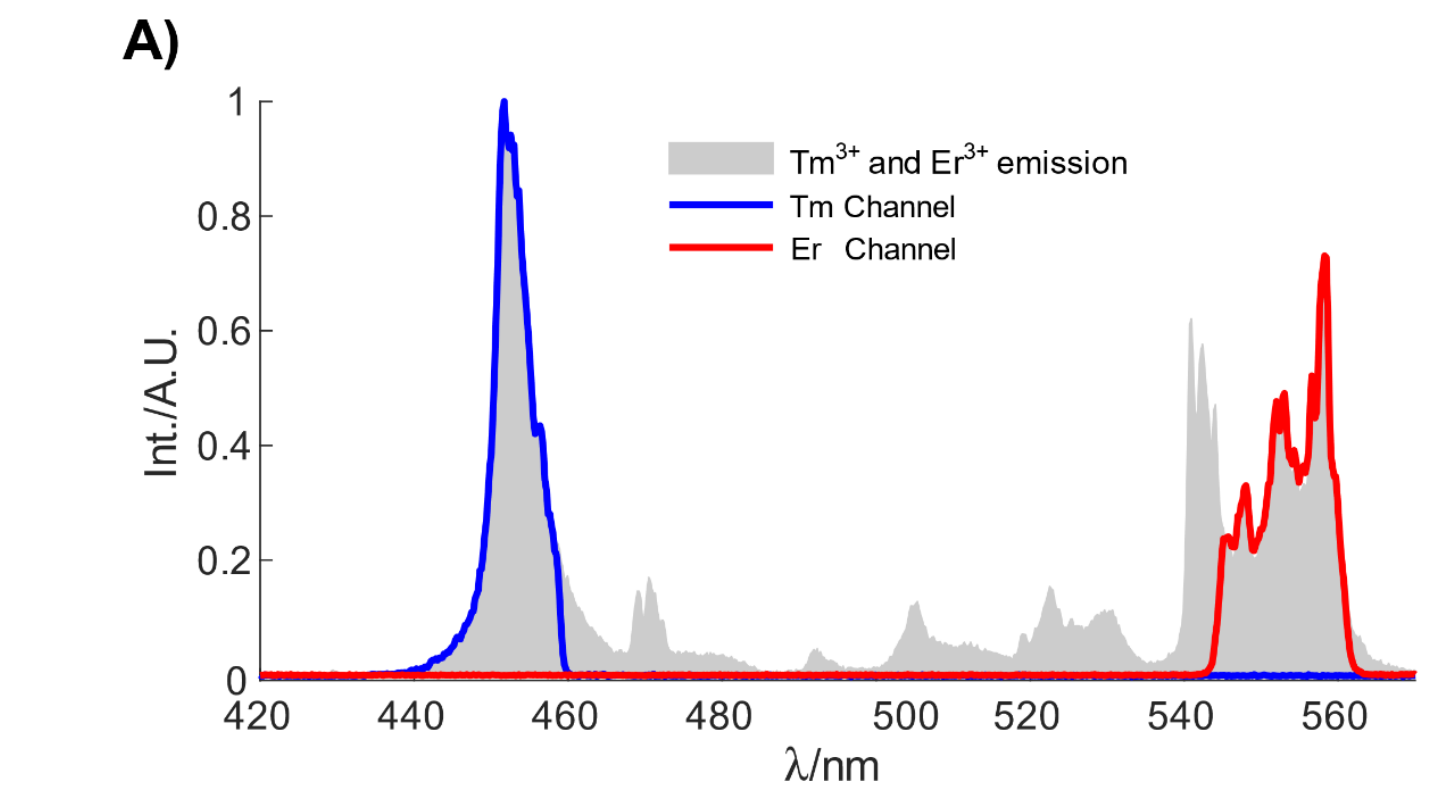

B)

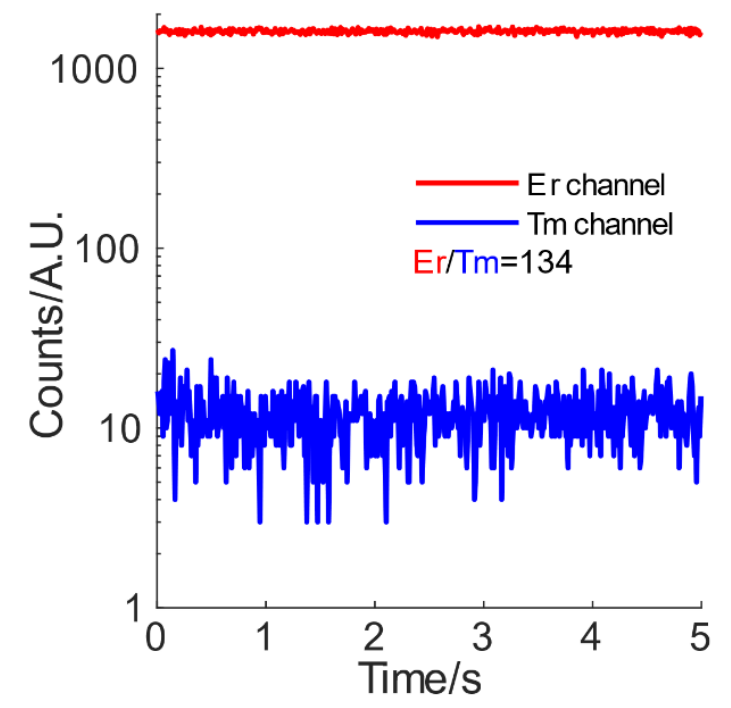

C)

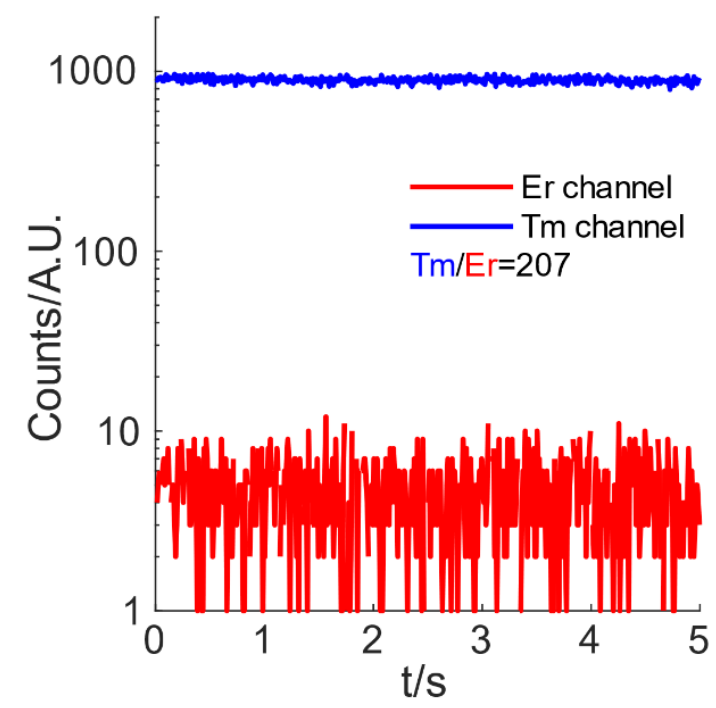

Figure S4. Cross-talk measurements. A) Region shown in grey corresponds to the spectrum obtained for a solution containing $\operatorname{Tm}(8 \%)$ and $\operatorname{Er}(3 \%)$ particles without any optical filters. The region shown in blue corresponds to the spectral region being probed for the Tm channel, and the red region corresponds to the Er channel. The "Tm and Er channel" spectra are obtained from the same solution but with the proper filter settings. A subsequent normalization is performed for both the Tm and Er channel such that the intensity of the individual channels overlay that of the combined emission spectrum (grey area). B) Intensity time trace measuring both the Er and Tm channel on a dried droplet from the $\operatorname{Er}(3 \%)$ stock solution with dark counts removed from the traces. A ratio between the average intensity of the Er channel compared to the Tm channel yields a ratio of 134. C) Similar to B), but now being performed on a dried droplet of $\operatorname{Tm}(8 \%)$ stock solution. This yields a ratio $(\mathrm{Tm} / \mathrm{Er})$ of 207. 

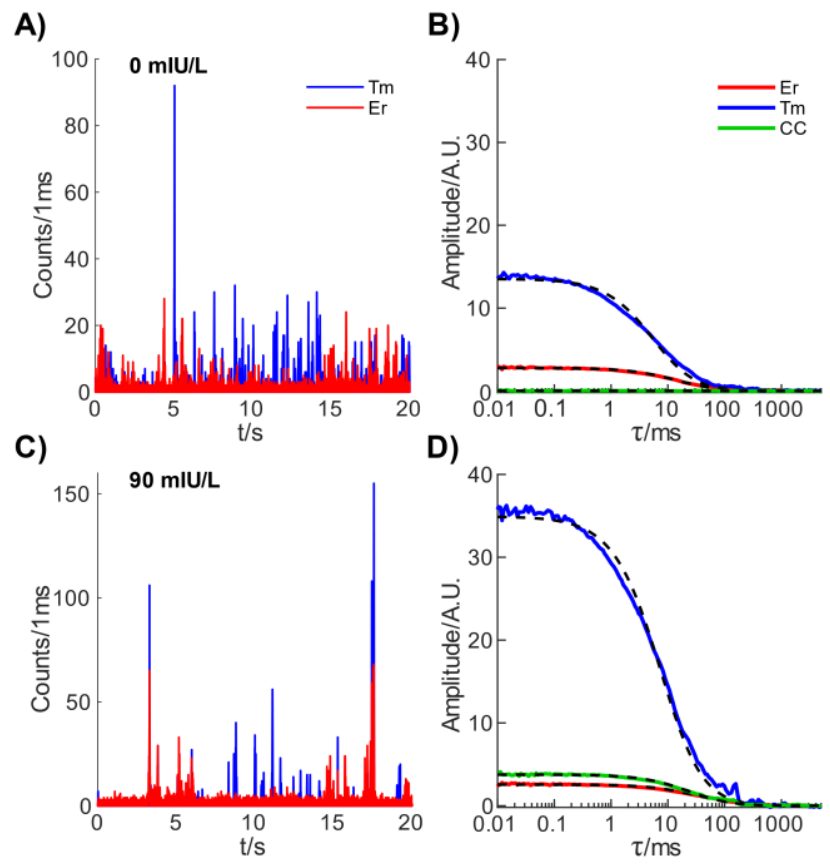

Figure S5. Section of a $300 \mathrm{~s}$ intensity time trace of $\operatorname{Tm}(8 \%)$ and $\operatorname{Er}(3 \%)$ particles (UCNP concentrations of $3.2 \mu \mathrm{g} / \mathrm{mL}$ ) in the TSH immunoassay A) in buffer solution without TSH and C) in buffer solution spiked with $90 \mathrm{mIU} / \mathrm{L} \mathrm{TSH}$. Corresponding auto-correlation functions of the $\operatorname{Er}(3 \%)$ and $\operatorname{Tm}(8 \%)$ as well as the cross-correlation functions can be seen in B) and D).
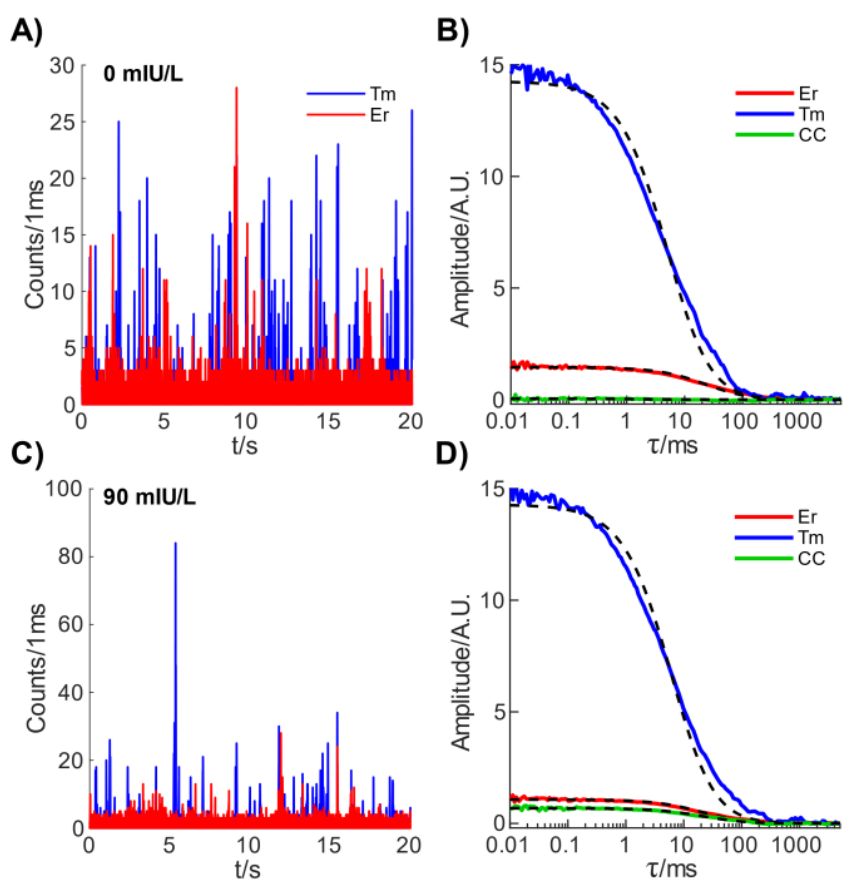

Figure S6. Section of a $300 \mathrm{~s}$ intensity time trace of $\operatorname{Tm}(8 \%)$ and $\operatorname{Er}(3 \%)$ particles (UCNP concentrations of $3.2 \mu \mathrm{g} / \mathrm{mL}$ ) in the THS immunoassay A) in a plasma solution without TSH and $\mathrm{C}$ ) in a plasma solution spiked with $90 \mathrm{mIU} / \mathrm{L}$ TSH. Corresponding auto-correlation functions of the $\operatorname{Er}(3 \%)$ and $\operatorname{Tm}(8 \%)$ as well as the cross-correlation functions can be seen in B) and D). 


\section{Upconversion cross-correlation immunoassay data analysis}

Auto- and cross-correlation of the acquired data was performed with the Burst Analyzer 2 software (Becker \& Hickl). Some treatment of the intensity time traces prior to correlating the data was performed in order to negate the effect of bright cross-linked species, which would otherwise affect the correlation curve. To remedy this issue, two different approaches were investigated for the high and low UNCP concentration series; either removing the obstructing cross-linked emission manually or by setting a threshold value. Deciding upon which features to remove manually was based on the relative brightness of the emissive species and the resulting auto- or cross-correlation curve, i.e. the appearance of shoulders at large time delays, $\tau$, indicate the existence of slowly diffusing cross-linked species. The fitting of auto- and crosscorrelation curves for the data treated with a manual removal was done in Burst Analyzer 2. The threshold approach necessitates deciding upon a certain value for a given binning time. For the first buffer series, where the high particle concentration results in high count rates, threshold values for either the Er or Tm channel of 400, 300, and 200 counts were used for a binning time of $1 \mathrm{~ms}$. For the second buffer series, threshold values of 200, 150, and 100 counts for binning times of $1 \mathrm{~ms}$ are instead used due to the lower count rates. For both series and channels a flank level of 50 counts was used. The flank level defines the count level an intense burst has to reach before it is no longer excluded from the time trace. Fitting of auto- and crosscorrelation curves treated with a threshold was done in MATLAB.

The effect of removing the luminescent bursts due to cross-linked species can be seen for the high UCNP concentration ( $72 \mu \mathrm{g} / \mathrm{mL}$ ) in buffer in Figure S7. Large intensity bursts can be seen in the time trace in Figure S7A, and upon examining the resulting auto- and cross-correlation curves when no data treatment is applied, it is obvious from the shoulders at around $500 \mathrm{~ms}$ that these bursts are due to large cross-linked species. Most of the features from the crosslinked species in the correlation curves are excluded upon removal of the bright bursts by a manual or threshold approach. Auto- and cross-correlation amplitudes are extracted from fits to a simple $3 \mathrm{D}$ diffusion model according to equation $(1)^{2}$ at the investigated range of TSH concentrations $(0-90 \mathrm{mIU} / \mathrm{L})$ under the different data treatment conditions; Figure S8 and Figure S9 show the cross- and auto-correlation amplitudes as a function of the TSH concentration.

$$
G(\tau)=A_{C C} \cdot\left(1+\frac{\tau}{\Delta \tau}\right)^{-3 / 2}
$$

While no data treatment yields significantly different results, there are only minor differences between the results obtained when treating the data manually or with a threshold. This is also reflected in the obtained LoDs given in Table S2. Based on these results, a threshold value of 400 counts was used for the preparation of the standard curve and exemplary auto- and crosscorrelation curves for the high UNCP concentration in buffer and plasma shown in the main article, though deciding upon a particular threshold value appears to have little bias on the results. 
Table S2. Limit of detections of TSH in UCCS immunoassay for high UCNP concentrations $(72 \mu \mathrm{g} / \mathrm{mL})$ in buffer obtained by treating the data at different conditions.

\begin{tabular}{llllll}
\hline Approach & None & Manual removal & 400 counts & 300 counts & 200 counts \\
\hline LoD $(\mathrm{mIU} / \mathrm{L})$ & 44.1 & 3.3 & 1.7 & 1.6 & 2.0 \\
\hline
\end{tabular}

A similar analysis was conducted for the low UCNP concentration $(3.2 \mu \mathrm{g} / \mathrm{mL})$ in buffer. Figure S10 shows the effect of removing emission from cross-linked species upon the resulting correlation functions, with similar results as for the high UCNP concentration buffer series. Cross- and auto-correlation amplitudes as a function of TSH concentration under different data treatment conditions are shown in Figure S11 and Figure S12, respectively. The resulting LoDs can be seen in Table S3. While the no data treatment approach yields the best LoD, it is, however, still preferable to exclude any potential obstructing cross-linked species by a threshold, that might otherwise render a measurement unusable (see e.g., the large autocorrelation amplitude deviations of erbium). In the main article, a threshold of 200 counts was accordingly used for the analysis of the low UCNP concentration data in both buffer and plasma.

Table S3. Limit of detections of TSH in UCCS immunoassay for low UCNP concentrations $(3.2 \mu \mathrm{g} / \mathrm{mL})$ in buffer obtained by treating the data at different conditions.

\begin{tabular}{llllll}
\hline Approach & None & Manual removal & 200 counts & 150 counts & 100 counts \\
\hline LoD $(\mathrm{mIU} / \mathrm{L})$ & 0.21 & 0.24 & 0.32 & 0.36 & 0.45 \\
\hline
\end{tabular}


A)

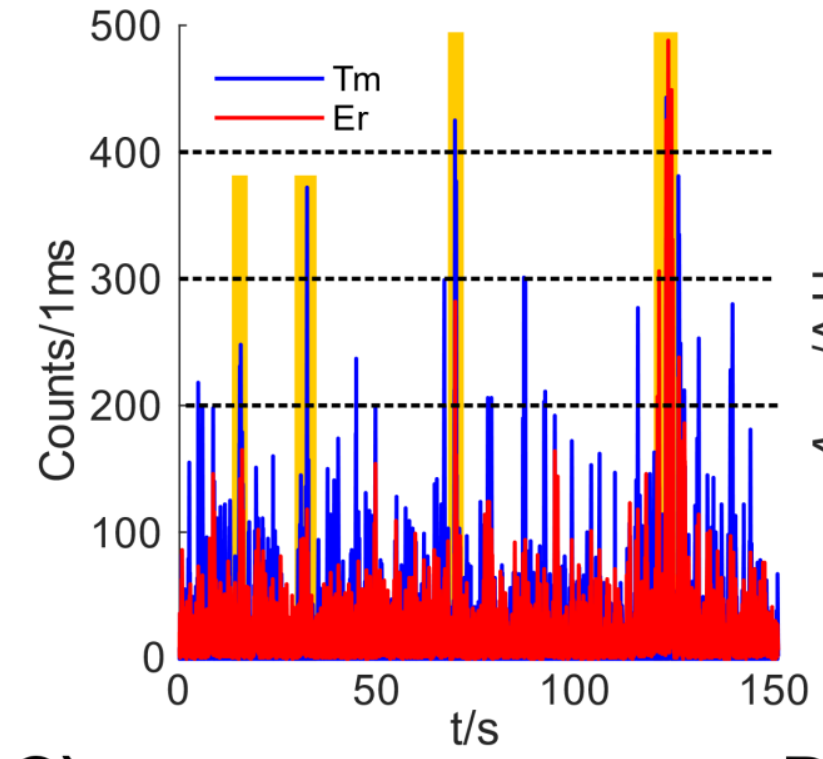

C)

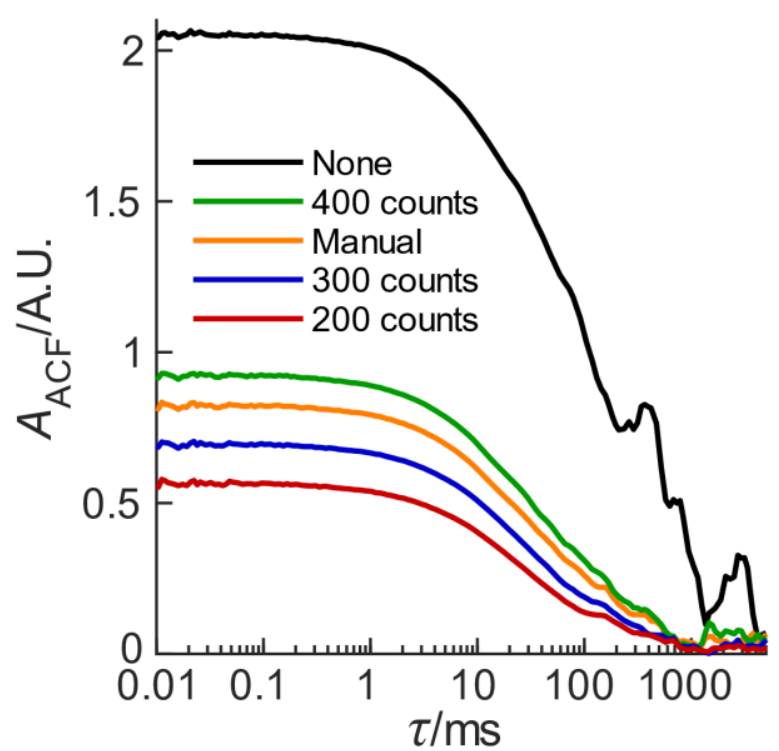

B)

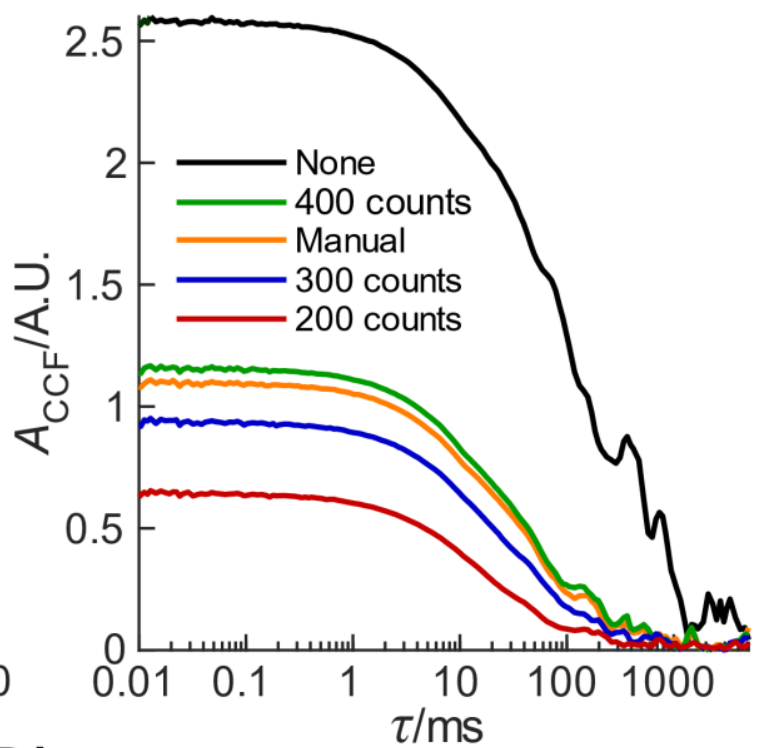

D)

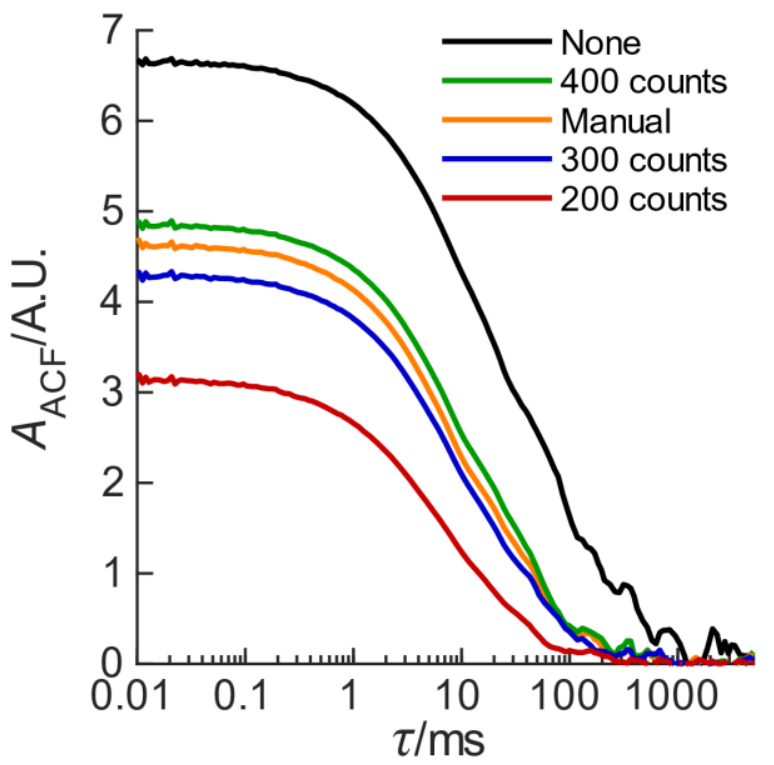

Figure S7. The effect of removing emission from cross-linked species upon the resulting correlation functions in the UCCS immunoassay at a high UCNP concentration $(72 \mu \mathrm{g} / \mathrm{mL})$. A) An intensity time trace from the $90 \mathrm{mIU} / \mathrm{L}$ TSH concentration showing the removal of data by defining a threshold (dashed lines) or by manually picking (yellow areas) out timespans with obstructing emission. B) Cross-correlation functions calculated from the intensity time traces in A) without any defined threshold, with a threshold of 400, 300, or 200 counts, or finally by manually removing emission stemming from cross-linked particles. C) Autocorrelation functions for the erbium channel from the time trace in A). D) Auto-correlation functions for the thulium channel from the time trace in A). 


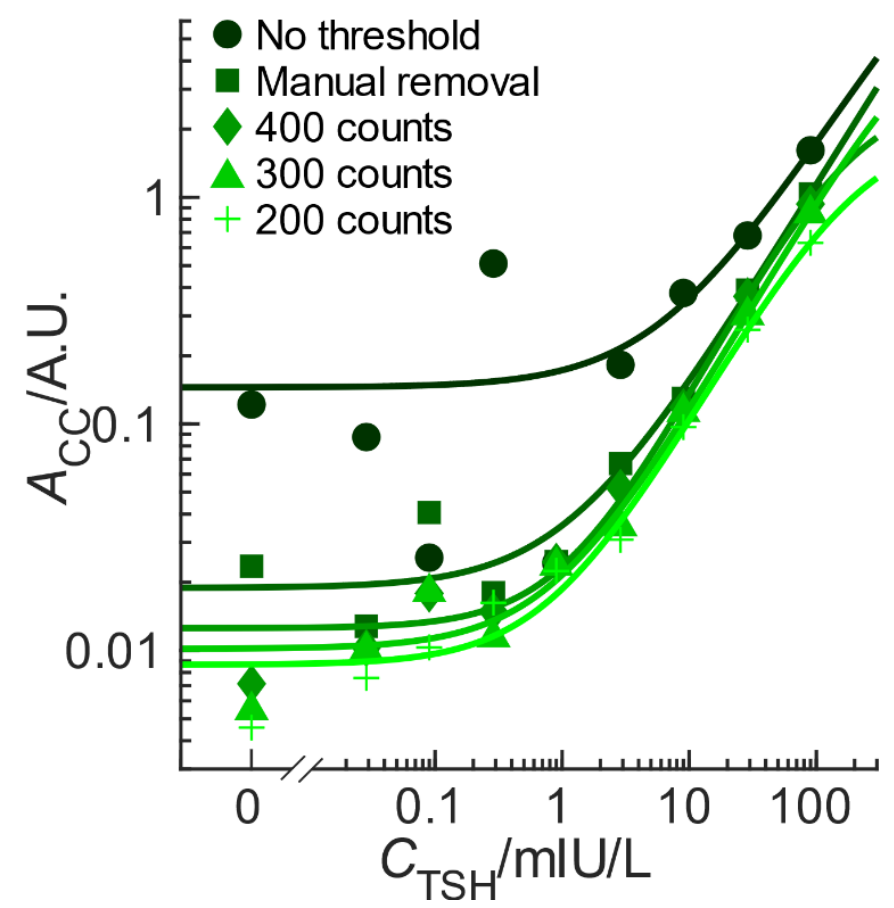

Figure S8. Standard curves for the TSH immunoassay at a high UCNP concentration ( $72 \mu \mathrm{g} / \mathrm{mL}$ ) resulting from different data treatment approaches. The $A_{\mathrm{CC}}$ values represent the average of six replicate measurements.

A)

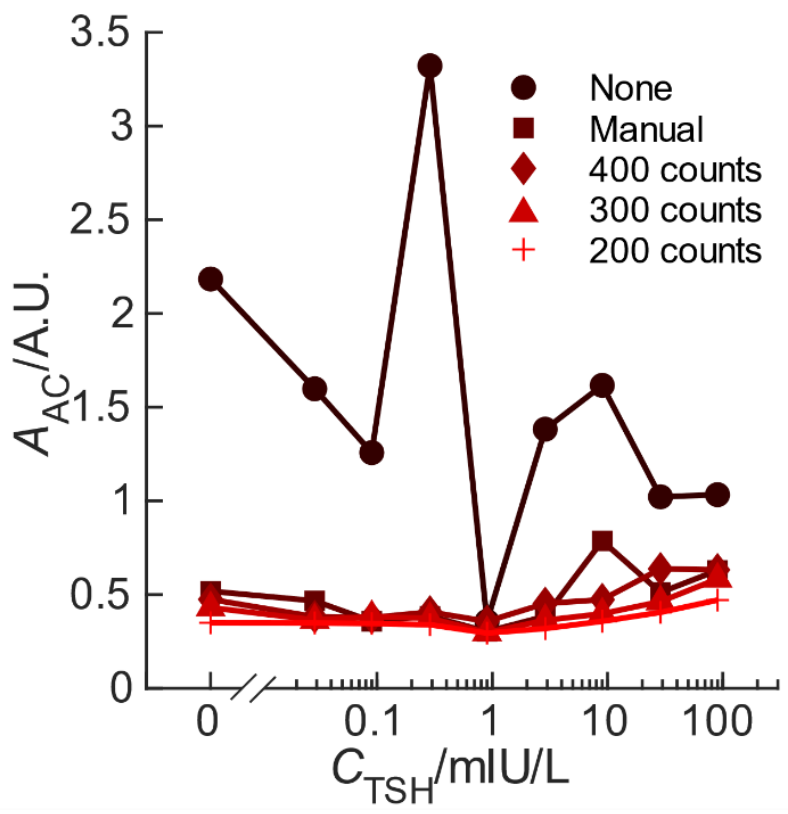

B)

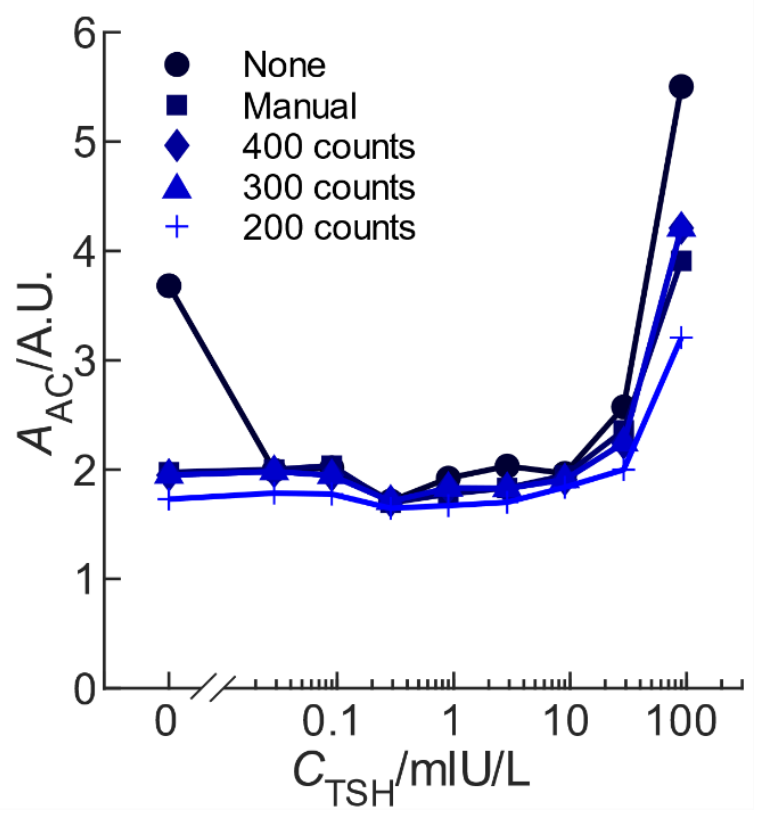

Figure S9. Amplitude of the auto-correlation function of A) erbium and B) thulium at different analyte concentrations and at a high UCNP concentration $(72 \mu \mathrm{g} / \mathrm{mL})$ in the TSH immunoassay. The $A_{\mathrm{AC}}$ values represent the average of six replicate measurements. There are large deviations when no threshold is used, while only minor differences are observed when a certain threshold is used. 
A)
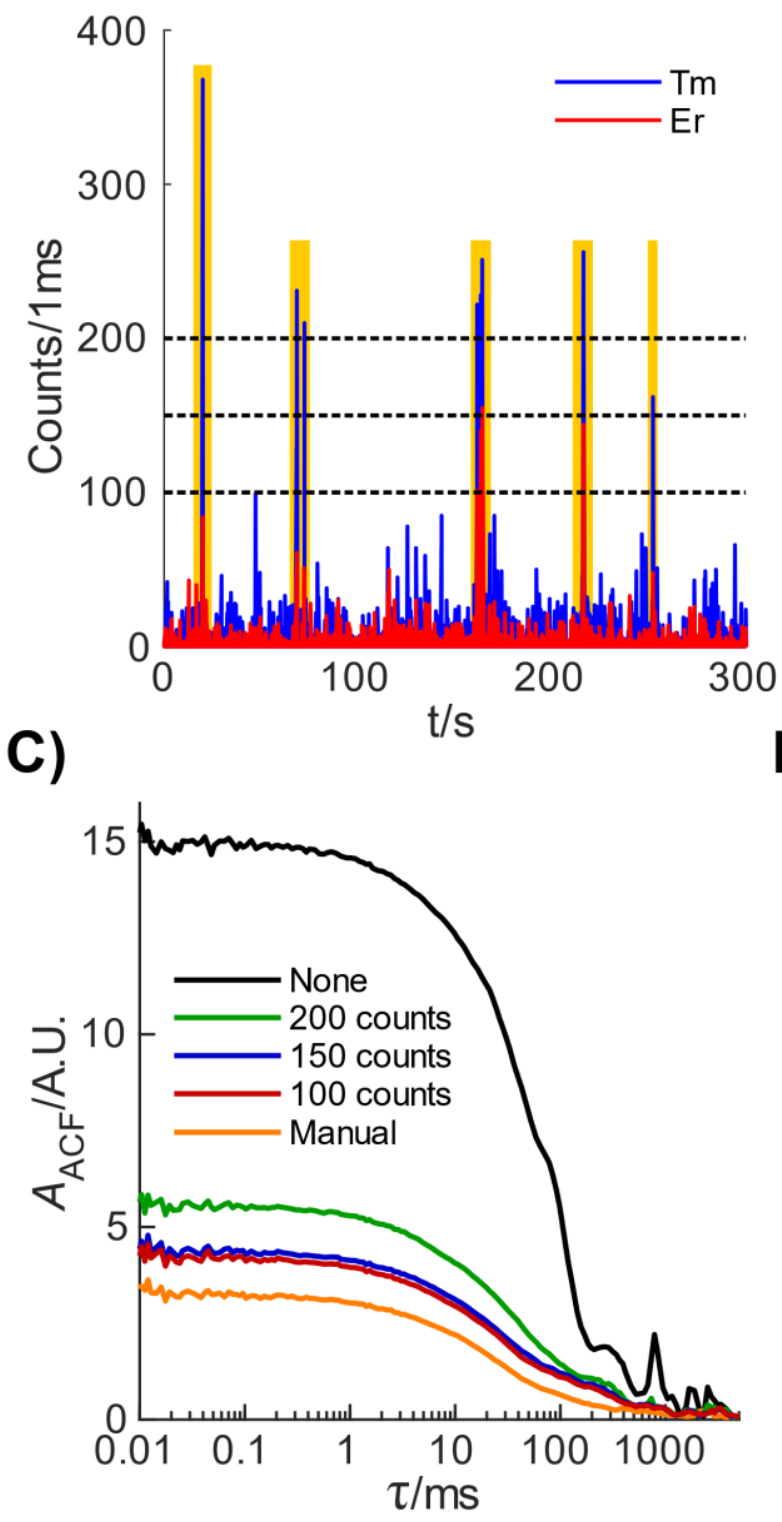

B)

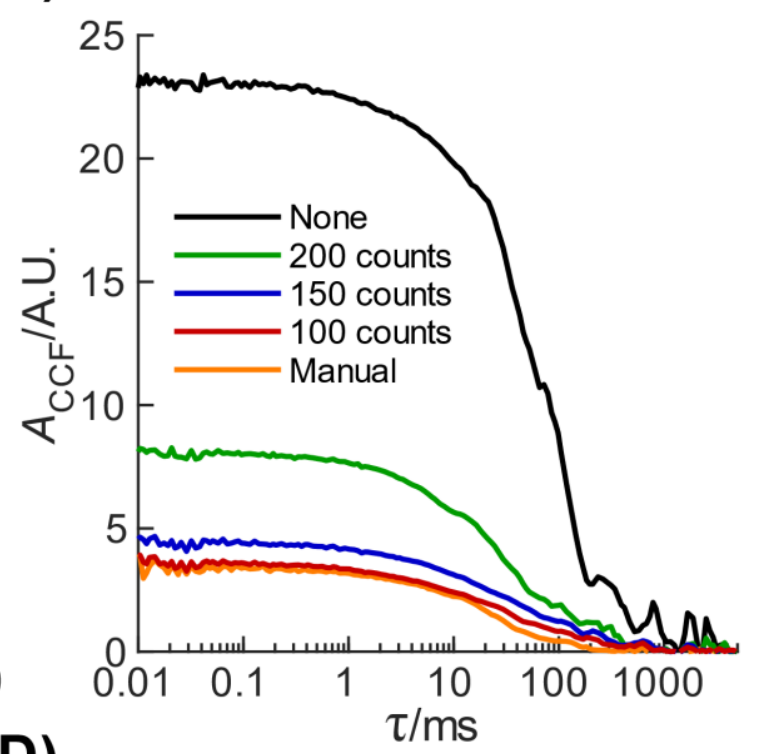

D)

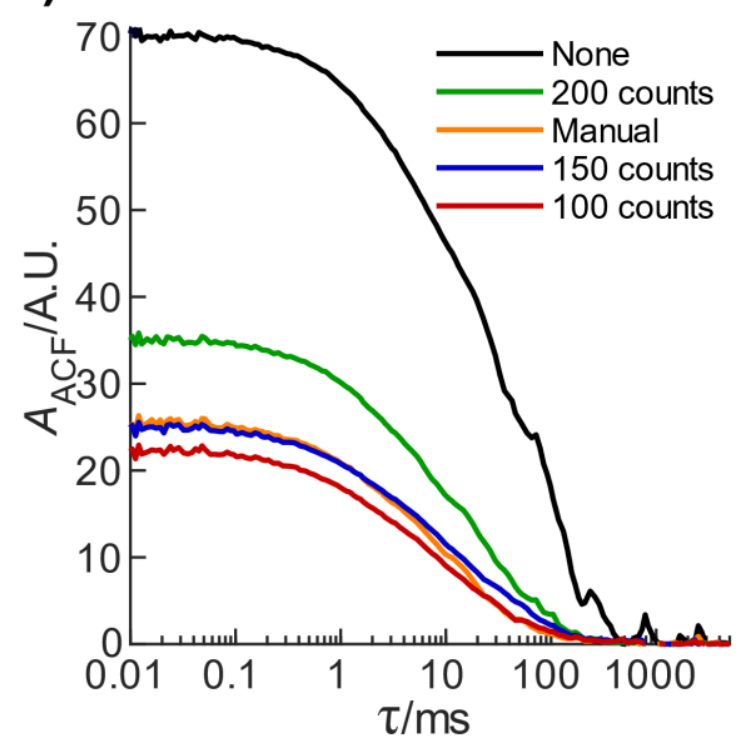

Figure S10. The effect of removing emission from cross-linked species upon the resulting correlation functions in the UCCS immunoassay at a low UCNP concentration $(3.2 \mu \mathrm{g} / \mathrm{mL})$. A) An intensity time trace from the $90 \mathrm{mIU} / \mathrm{L}$ TSH concentration showing the removal of data by defining a threshold (dashed lines) or by manually picking (yellow areas) out timespans with obstructing emission. B) Cross-correlation functions calculated from the intensity time traces in A) without any defined threshold, with a threshold of 200,150, or 100 counts, or finally by manually removing emission stemming from cross-linked particles. C) Autocorrelation functions for the erbium channel from the time trace in A). D) Auto-correlation functions for the thulium channel from the time trace in A). 


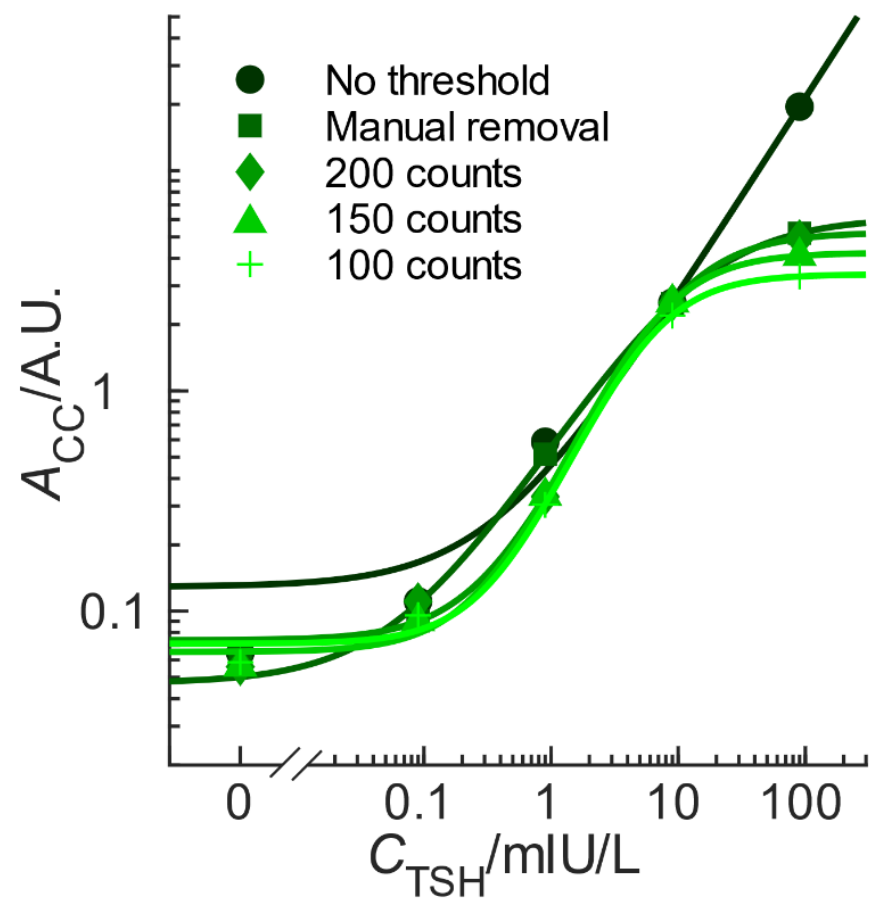

Figure S11. Standard curves for the TSH immunoassay at a low UCNP concentration (3.2 $\mu \mathrm{g} / \mathrm{mL})$ resulting from different data treatment approaches. The $A_{\mathrm{CC}}$ values represent the average of six replicate measurements.

A)

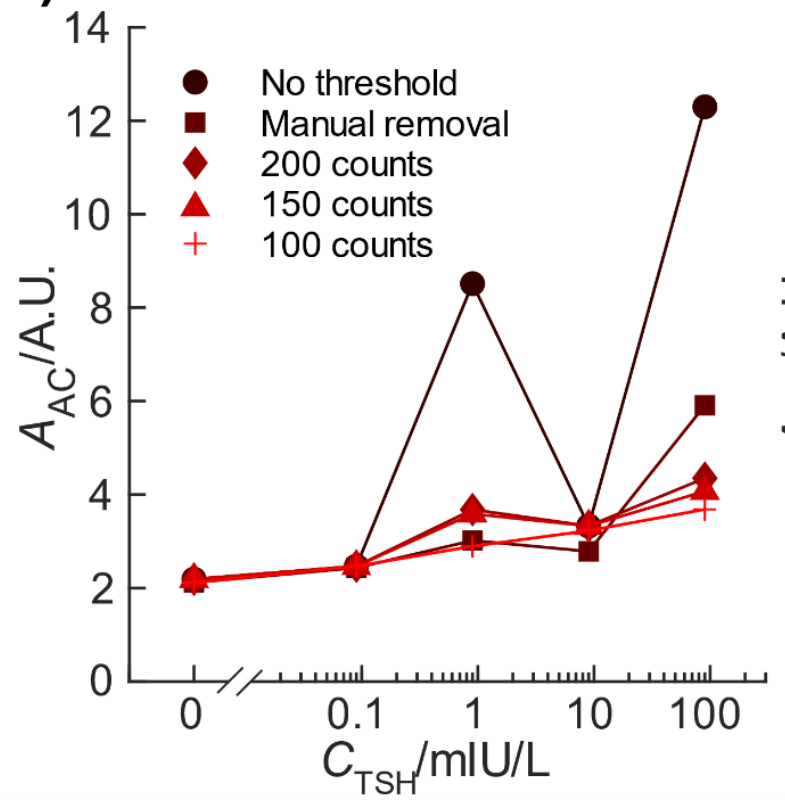

B)

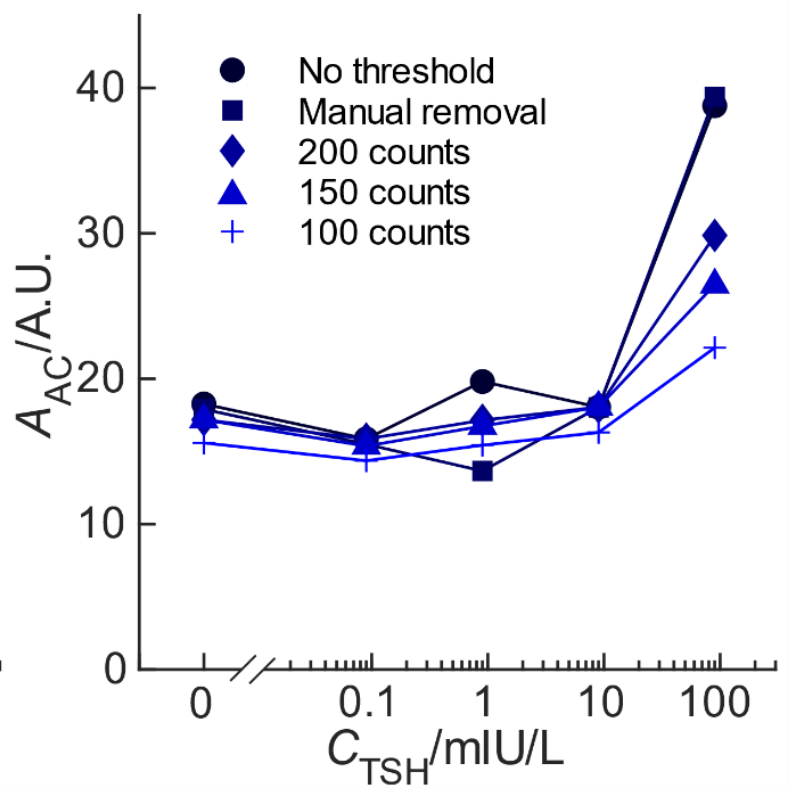

Figure S12. Amplitude of the auto-correlation function of A) erbium and B) thulium at different analyte concentrations and at a low UCNP concentration $(3.2 \mu \mathrm{g} / \mathrm{mL})$ in the TSH immunoassay. There are significant deviations when no threshold is used, while only minor differences are observed when a certain threshold is used. 
Table S4. Fitting parameters of the four-parameter logistic functions used to fit the TSH immunoassay standard curves in buffer and plasma with high $(72 \mu \mathrm{g} / \mathrm{mL})$ and low $(3.2 \mu \mathrm{g} / \mathrm{mL})$ UCNP concentrations. Errors are given as $95 \%$ confidence intervals.

\begin{tabular}{lllll}
\hline & \multicolumn{1}{c}{$\mathrm{A}_{1}$} & \multicolumn{1}{c}{$\mathrm{A}_{2}$} & \multicolumn{1}{c}{$\mathrm{c}_{0}(\mathrm{mIU} / \mathrm{L})$} & $\mathrm{p}$ \\
\hline Buffer (high UCNP) & $2.91( \pm 1.64)$ & $0.013( \pm 0.006)$ & $183.1( \pm 158.8)$ & $1.07( \pm 0.15)$ \\
Plasma (high UCNP) & $3.08( \pm 22.74)$ & $0.007( \pm 0.019)$ & $667.8( \pm 7241)$ & $0.91( \pm 0.64)$ \\
Buffer (low UCNP) & $5.24( \pm 0.83)$ & $0.007( \pm 0.227)$ & $9.84( \pm 3.86)$ & $1.23( \pm 0.82)$ \\
Plasma (low UCNP) & $0.82( \pm 0.33)$ & $0.083( \pm 0.167)$ & $8.22( \pm 7.33)$ & $1.82( \pm 10.00)$ \\
\hline
\end{tabular}
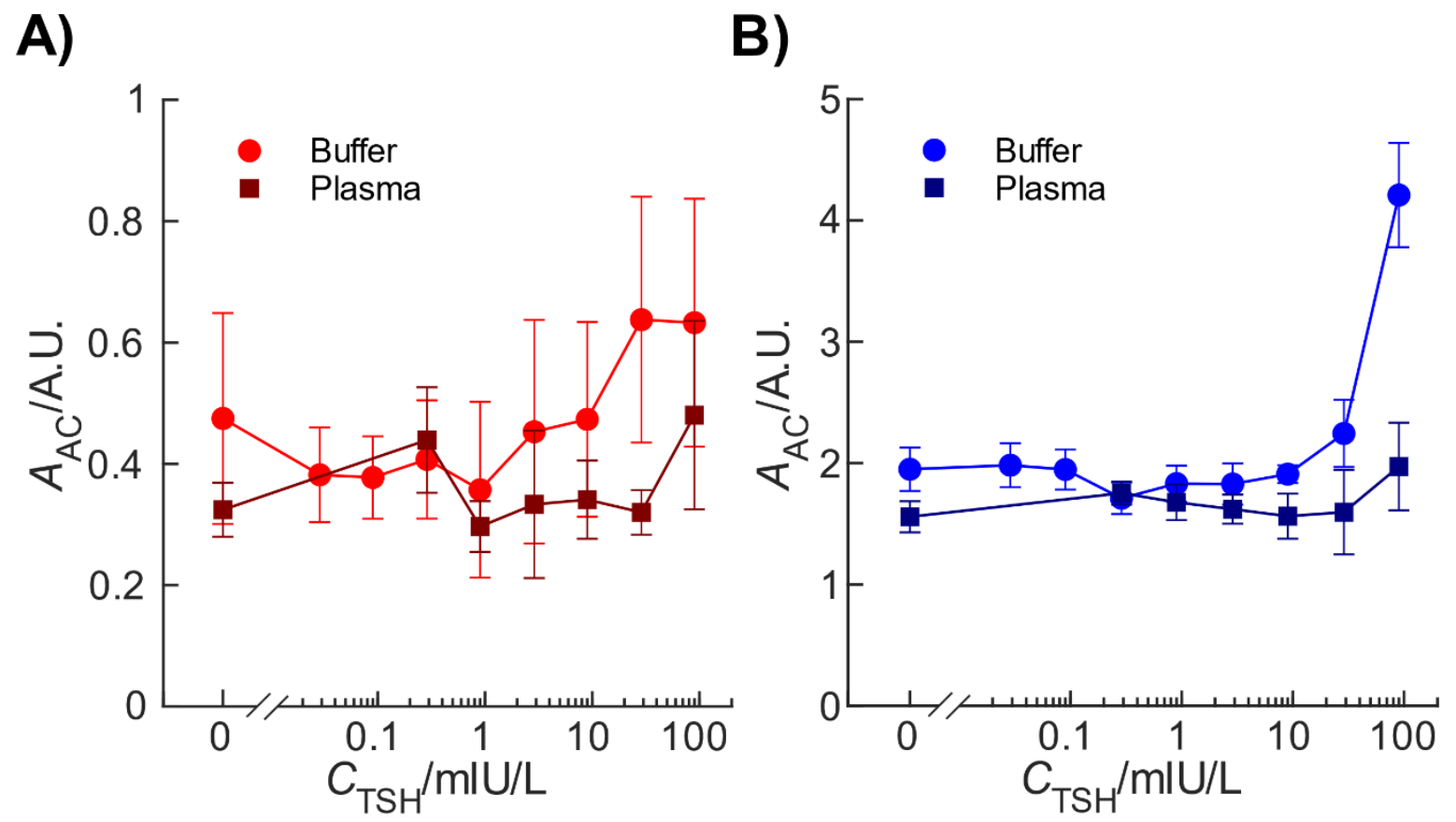

Figure S13. Amplitude from the ACFs of A) $\operatorname{Er}(3 \%)$ and B) $\operatorname{Tm}(8 \%)$ in buffer and plasma at a high UCNP concentration $(72 \mu \mathrm{g} / \mathrm{mL})$ in the TSH immunoassay. The $A_{\mathrm{AC}}$ values and their error bars represent the averaged amplitudes and standard deviations of six replicate measurements. Measurements were conducted for $150 \mathrm{~s}$. 


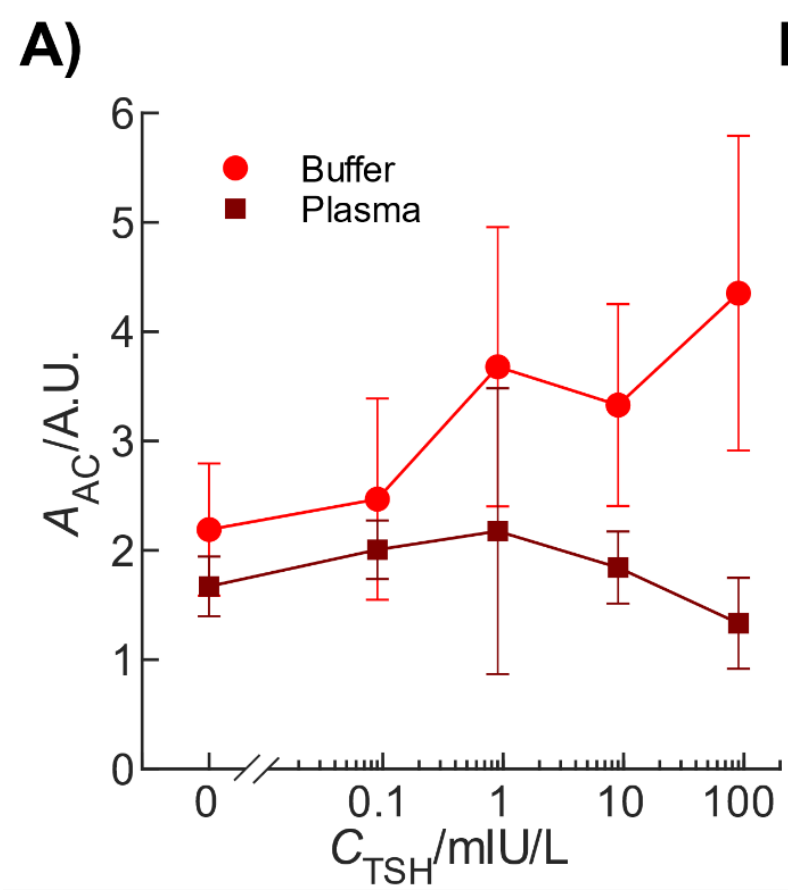

B)

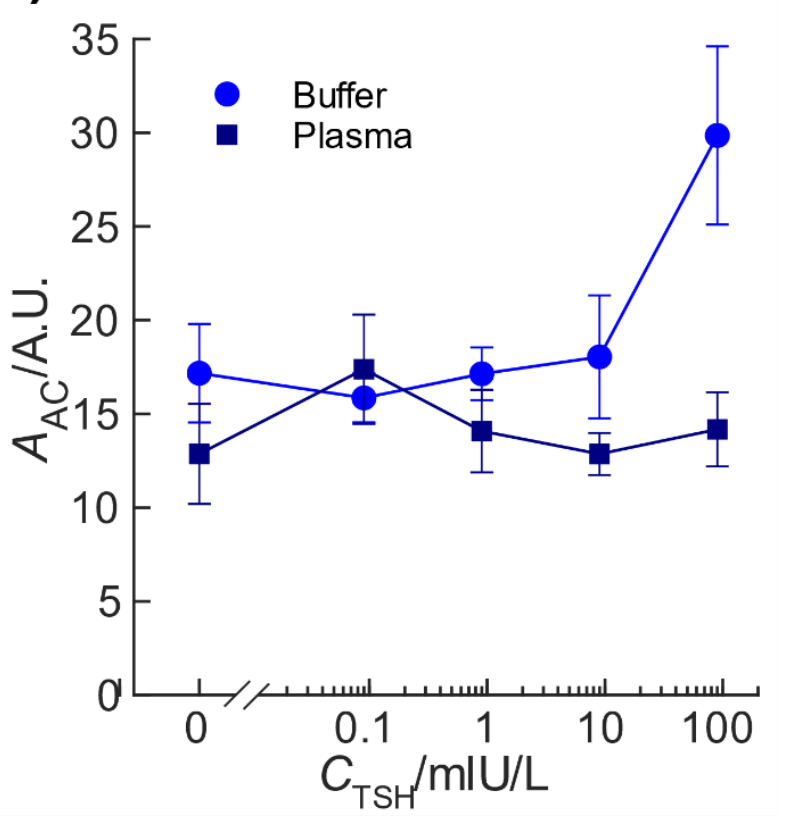

Figure S14. Amplitude from the ACFs of A) $\operatorname{Er}(3 \%)$ and B) $\operatorname{Tm}(8 \%)$ in buffer and plasma at a low UCNP concentration $(3.2 \mu \mathrm{g} / \mathrm{mL})$ in the TSH immunoassay. The $A_{\mathrm{AC}}$ values and their error bars represent the averaged amplitudes and standard deviations of six replicate measurements. Measurements were conducted for $300 \mathrm{~s}$.

\section{References}

1. Lahtinen, S.; Krause, S.; Arppe, R.; Soukka, T.; Vosch, T., Upconversion Cross-Correlation Spectroscopy of a Sandwich Immunoassay. Chem. - Eur. J. 2018, 24 (37), 9229-9233.

2. Hess, S. T.; Huang, S.; Heikal, A. A.; Webb, W. W., Biological and Chemical Applications of Fluorescence Correlation Spectroscopy: A Review. Biochemistry 2002, 41 (3), 697-705. 